\title{
Objective Classification of Precipitating Convective Regimes Using a Weather Radar in Darwin, Australia
}

\author{
SIMON CAINE \\ Monash University, Melbourne, Victoria, Australia \\ CHRISTIAN JAKOB \\ Bureau of Meteorology Research Centre, Melbourne, Victoria, Australia \\ STEVEN SIEMS \\ Monash University, Melbourne, Victoria, Australia \\ PETER MAY \\ Bureau of Meteorology Research Centre, Melbourne, Victoria, Australia
}

(Manuscript received 17 January 2008, in final form 16 September 2008)

\begin{abstract}
A clustering algorithm was applied to Frequency with Altitude Diagrams (FADs) derived from 4 yr of hourly radar data to objectively define four tropical precipitation regimes that occur during the wet season over Darwin Australia. The precipitation regimes defined are distinguished in terms of convective intensity, presence of stratiform precipitation, and precipitation coverage. Regime 1 consists of patchy convection of medium intensity and low area coverage, and regime 2 contains strong convection with relatively small area coverage. Regime 3 is composed of weak convection with large area coverage and large stratiform regions, and regime 4 contains strong convection with large area coverage and large stratiform regions. Analysis of the seasonal cycle, diurnal cycle, and regime occurrence as a function of monsoon activity all provide insight into the different physical character of the precipitation regimes. Two of the regimes exhibit a diurnal cycle with a peak in the afternoon, while the other two show a peak in their frequency of occurrence in the early morning. The different character of the regimes is also confirmed by the varying contributions that convective and stratiform rainfall make to the overall within-regime precipitation.
\end{abstract}

\section{Introduction}

Tropical rainfall is largely controlled by convective processes. However, as has been shown in numerous studies (e.g., Orlanski 1975; Rickenbach and Rutledge 1998; Nesbitt et al. 2000, 2006; Houze et al. 1981; Houze and Churchill 1984; Houze 1987; Mapes and Houze 1993; Mapes 1993; Houze et al. 1980), these processes are often organized on a variety of scales, that can vary from the local to mesoscale (e.g., topographically driven convection) to the large scale in convectively coupled

Corresponding author address: Simon Caine, School of Mathematical Sciences, Monash University, 3800 Melbourne, VIC, Australia.

E-mail: simon.caine@sci.monash.edu.au tropical waves (e.g., the Madden-Julian oscillation). Because of convective organization, the spatial and temporal structure of tropical precipitation events is far more complex than that of a simple archetypal convective shower. Many convective systems exhibit a large stratiform component (Houze 1997, 1993), identifiable both by its anvil cloud signature and its spatially extensive but lower (in comparison to a convective shower) rain rates. The stratiform component of tropical rainfall constitutes a major fraction of the total both globally and regionally (Schumacher and Houze 2003) and its vertical structure of latent heat release is likely to play an important role in the interaction of convection with large-scale dynamical features of the tropical atmosphere (Lin et al. 2004; Schumacher et al. 2004; Mapes and Lin 2005; Tao et al. 2006). Recently, Mapes et al. 
(2006) have shown that the archetypal composition of precipitating tropical convective systems, consisting of a shallow and deep convective mode, as well as a stratiform mode, is observable over many scales. This raises the question, if convective systems in the tropics indeed occur in distinguishable and repeatedly occurring regimes, what are the characteristics of those regimes and how are they linked to the well-known tropical circulation features?

Jakob and Tselioudis (2003) and Rossow et al. (2005) used a simple clustering algorithm applied to data from the International Satellite Cloud Climatology Project (ISCCP; Schiffer and Rossow 1983), to show that tropical cloudiness on a $280 \times 280 \mathrm{~km}^{2}$ scale (the size of the ISCCP grid box) appears to be organized into six major regimes: three convectively active (and hence likely precipitating) and three suppressed. By their very nature the regimes identified in this fashion are based on the radiative signature of the tropical cloud systems, and further work (Jakob et al. 2005) established that the ISCCP-based regimes show distinct signatures in their radiative and thermodynamic properties. However, because of a lack of suitable data no conclusions on the precipitation characteristics of the ISCCP-based regimes could be drawn. Nevertheless, based on the success of these studies it is valid to ask the question if a similar regime character can be found in the precipitation signature of tropical convection.

An ideal tool to observe the spatial structure of convective cloud systems is the centimeter wavelength radar. Previously Boccippio et al. (2005) used clustering analysis on vertical profiles derived from the Tropical Rainfall Measuring Mission (TRMM) precipitation radar to define a number of archetypal vertical structures for the entire tropics. The profiles used by Boccippio et al. (2005) were essentially vertical columns with a horizontal diameter of $4 \mathrm{~km}$, consequently very little spatial information entered into their regime definition.

Ground-based radars are routinely deployed by weather services worldwide (Demott and Rutledge 1998; Kawashima et al. 2006) for research and forecasting purposes. The Australian Bureau of Meteorology has operated a polarimetric research C-band radar (C-Pol; Keenan et al. 1998; May et al. 1999; Carey and Rutledge 2000), in Darwin (Australia) since 1995. Darwin is located in the tropical north of Australia and has been the site of numerous field studies on tropical convection, such as the Australian Monsoon Experiment (AMEX; Holland et al. 1986; Gunn et al. 1989; Keenan et al. 1989), the Marine Continental Thunderstorm Experiment (MCTEX; Keenan et al. 2000; Carbone et al. 2000), the Darwin Area Wave Experiment (DAWEX; Hamilton et al. 2004; Alexander et al. 2004; Pautet et al. 2005), and most recently the Tropical Warm Pool In- ternational Cloud Experiment (TWP-ICE; May et al. 2005). These experiments established that with its tropical monsoon climate, characterized by distinct wet, dry, and transition seasons, Darwin constitutes an ideal location to observe many of the variety of convective systems observed in the tropics (Keenan and Carbone 1992; Keenan and Rutledge 1993). During active monsoon spells, convection in the Darwin area has been shown to often resemble maritime convection found over the tropical oceans (Steiner et al. 1995; Keenan and Carbone 1992). The often topographically driven convective systems observed during both the transition seasons and in monsoon breaks on the other hand are among the most intense observed on the planet (Crook 2001; Zipser et al. 2006) and have been shown to be good examples of continental convection observed over the tropical continents. For these reasons the Atmospheric Radiation Measurement (ARM) program (Ackerman and Stokes 2003) established the third Tropical Western Pacific (TWP) climate research facility in Darwin. The combination of the availability of many years of radar data and the ability to observe a variety of convective systems makes Darwin an ideal location to study the possible regime character of tropical precipitation on scales of a few hundred kilometers.

The aim of this paper is to determine if such regimes can be objectively defined from radar data and used to describe the rainfall-precipitation properties of convective systems occurring over long periods of time (e.g., seasons) for the region surrounding Darwin. To establish if tropical rainfall exhibits such regime character this study applies a cluster algorithm similar to that used in Jakob and Tselioudis (2003) and Rossow et al. (2005) to Frequency with Altitude diagrams (FADs; Yuter and Houze 1995; Neiman et al. 2005) of radar reflectivity derived from four wet seasons of radar data. The properties of the regimes identified by this algorithm are investigated and their relationship to the main circulation regime in tropical Australia-the monsoonis established. The relationship of the radar-based regimes to the ISCCP-based tropical cloud regimes is also investigated.

\section{Data description and method}

The dataset used in this study is composed of reflectivity values observed by the scanning C-band polarimetric (C-Pol) radar located near Darwin (Keenan et al. 1998). Figure 1 shows a map of the radar domain indicating land areas (gray) and the maximum coverage of the radar footprint (achieved at $3 \mathrm{~km}$ in the vertical) used in this study. The radar performs a full volume scan every $10 \mathrm{~min}$. Given the aim of studying possible 


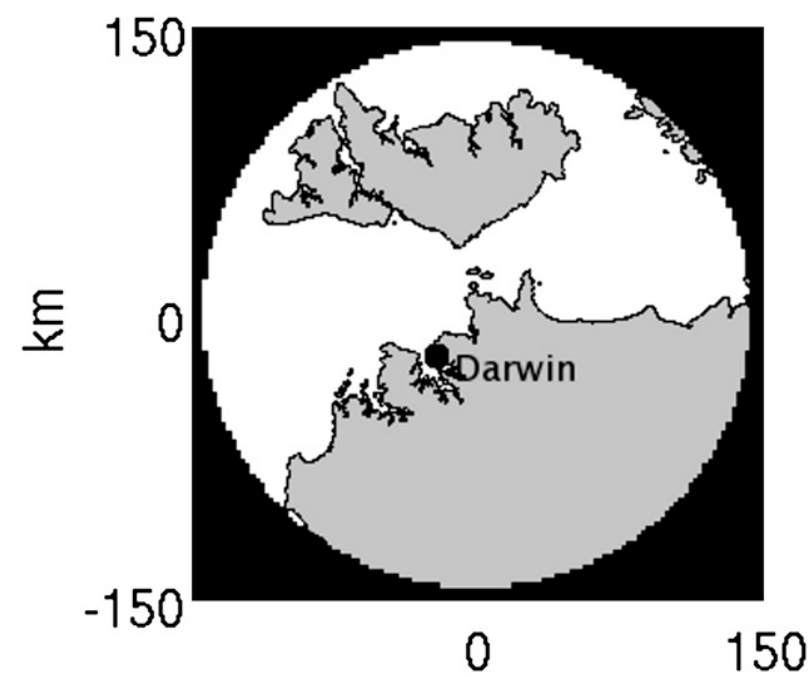

FIG. 1. CAPPI showing the maximum coverage of the Gunn Point radar, near Darwin, Australia. CAPPI is valid at $3 \mathrm{~km}$ in the vertical. The land is shaded gray and the oceans are white.

precipitation regimes as they occur over long periods of time it was necessary (because of computational limitations) to reduce the volume of information by using only one scan per hour. The hourly dataset used in this investigation is composed of the four wet seasons [November-April (NDJFMA)] of 1999/2000, 2001/02, $2002 / 03$, and $2003 / 04$, bringing the total number of sampled radar volumes to just over 13500 .

As a first step in calculating FADs (Yuter and Houze 1995) of radar reflectivity for each of the 13500 sampled volumes, the volume scans were interpolated into a Cartesian space consisting of 40 vertical levels with a grid spacing of $500 \mathrm{~m}$, and 121 grid points in each horizontal direction with a grid spacing of $2.5 \mathrm{~km}$. The change in the horizontal extent of the radar domain with height is taken into account by applying masks of the actual observed area at each vertical level. The histograms forming the basis of the FADs were then created by iteratively searching through all valid reflectivity values at each vertical level and calculating the frequency with which these values fall into reflectivity bins of width $2 \mathrm{dBZ}$. Reflectivity values below $0 \mathrm{~dB} Z$ are excluded from the analysis as they are considered at the detection limit of the radar, especially at longer ranges. At each vertical level the relative frequency of occurrence of each reflectivity bin was then calculated by dividing the number in each bin by the total number of observed points defined by the radar mask at that level. Consequently, summation over the frequencies for each vertical level can be thought of as a measure for the fractional coverage with radar signal (or precipitation) at that level. This deliberately deviates from the stan- dard normalization of Contoured Frequency with Altitude Diagrams (CFADs, the graphical representation of FADs; Yuter and Houze 1995), where each level is normalized by its fractional coverage. This choice was made to maintain area coverage information in the regime analysis that follows, as this provides a crucial distinction between regimes.

For illustrative purposes Fig. 2 shows the mean FAD over all samples used in this study. An important summary measure used throughout the study is the total volume coverage (TVC) with radar echo. This quantity is calculated by summing the number of radar returns over all levels and reflectivity values then dividing by the total number of observable points. It therefore represents the fractional coverage of the radar signal over the entire three-dimensional domain. The TVC for the mean histogram is 0.04 , indicating that on average approximately $4 \%$ of the volume scanned by the radar has a reflectivity value above the $0-\mathrm{dB} Z$ threshold. It can also be seen that, on average, the maximum echotop height reaches approximately $16 \mathrm{~km}$ and reflectivity values greater than $40 \mathrm{dBZ}$ are observed up to $8 \mathrm{~km}$. A large fraction of the returns have reflectivity values less than $18 \mathrm{dBZ}$. As this is the cutoff threshold for the TRMM radar, Fig. 2 gives some indication of the number of hydrometeors the TRMM radar will not observe.

Each of the more than 13500 histograms provides an input into the definition of objectively derived regimes. To focus the clustering algorithm (see below) onto the major regimes, first all (trivial) null cases (i.e., those with little or no radar return anywhere in the volume) were removed from the analysis. For this purpose any histogram that did not have at least $1 \%$ coverage in at least one of the vertical levels was defined as a noprecipitation histogram and was removed. This eliminated roughly 5000 histograms from the analysis. While these "no-precipitation" cases do not enter the regime definition algorithm, they do nevertheless provide important physical information about the state of the atmosphere. Their high frequency of occurrence and their "trivial" nature justifies their removal to avoid them dominating the outcome of the cluster algorithm.

A K-means clustering algorithm (Anderberg 1973) was applied to the remaining histograms to identify possible recurring patterns in the FADs that could be indicative of recurring precipitation regimes. The choice of algorithm is largely driven by its successful application in previous studies (Jakob and Tselioudis 2003) and efficiency requirements due to the large sample size used here. As discussed in Jakob and Tselioudis (2003) and Rossow et al. (2005) a feature of this particular algorithm is that it requires the user to predefine the 


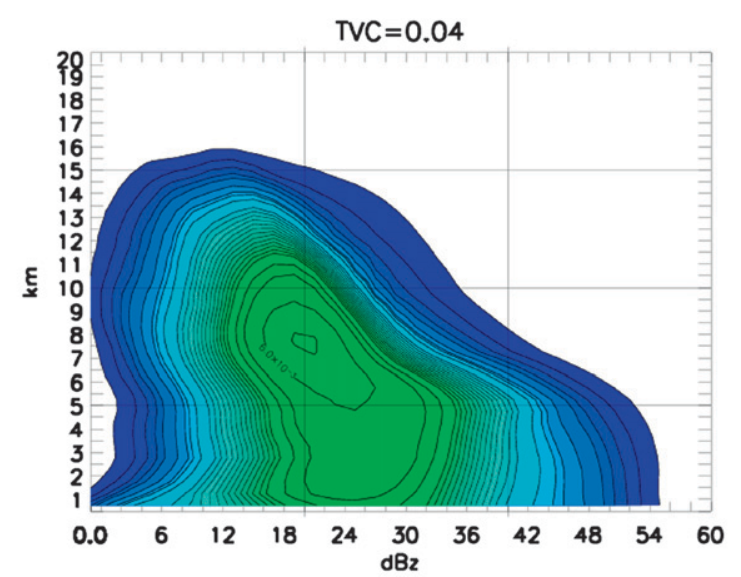

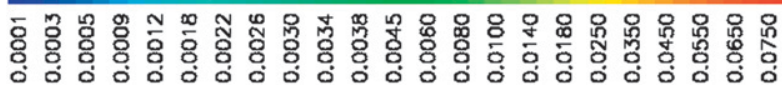

FIG. 2. FAD showing the mean of all time periods used in the study (13 508 histograms). The contours show bin counts divided by total observed grid points. The TVC is shown at the top of the diagram, indicating that on average $4 \%$ of the volume scanned by the radar is covered by hydrometeors.

number of clusters searched for (i.e., $k$ ). This is overcome by repeating the analysis several times with varying values of $k$. Here $2 \leq k \leq 10$ are used. The basic concept of the iterative $\mathrm{K}$-means algorithm is as follows:

- randomly choose $k$ "seed vectors" from the dataset as initial cluster centroids,

- assign each input histogram to one cluster by finding the minimum Euclidean distance to any of the cluster centroids,

- calculate new cluster centroids by averaging over all histograms assigned to a cluster,

- use the newly calculated centroids as seed vectors, and

- iterate the algorithm.

With each iteration the difference between the old and new centroids decreases and the clusters are said to be stable when this difference is less than a prescribed threshold value. The histograms associated with each cluster are known to be closer to the centroid of this cluster than to those of any of the others. Each regime is then characterized by its centroid or mean histogram. The regimes defined by the clustering algorithm should not depend on initial histograms chosen at random and tests were performed to ensure this was the case.

The advantage of using the K-means algorithm is that it provides an objective method for defining regimes from large sets of data. However, as discussed above, the number of clusters, $k$, searched for in the algorithm needs to be prescribed. It is therefore necessary to define a strategy for choosing the optimal set of clusters from the application of the algorithm to values of $2 \leq$ $k \leq 10$. Here the same "quasi-objective" method as in Rossow et al. (2005) is used. For each increase in cluster number the stability of the solution to the (randomly chosen) initial seeds is investigated. Furthermore, the emerging new cluster in the $k+1$ analysis is compared to the existing $k$ clusters of the previous application. The optimal number of regimes in this study, $K$, is then defined as the smallest possible number of clusters for which (i) the algorithm provides stable solutions with respect to initial seeds and (ii) the addition of further clusters leads to regimes similar to the already existing ones. Here it is found that $K=4$. Note, that since noprecipitation histograms have been excluded from the analysis to begin with, the total number of physical regimes defined in this study is five. Nevertheless, the rest of the paper will continue to refer to four precipitation regimes, treating the no-precipitation regime as a "zeroth" regime because of its trivial character. The main features of the four major precipitation regimes identified will now be described. It should be noted a particular storm event or mesoscale convective system (MCS) may and indeed does fall into multiple precipitation regimes. Transition of a storm event or MCS between regimes occurs when the temporal evolution of the event is captured within the domain of the radar. Transition between regimes may also occur when a storm event or MCS enters and leaves the domain of the radar.

\section{Tropical precipitation regimes as identified in radar data}

The application of a cluster algorithm to the FADs derived from hourly volume scans using the polarimetric CPol radar located near Darwin reveals four major precipitation regimes in that region. To determine the robustness of the precipitation regimes each wet season was analyzed separately. The regimes defined when using individual seasons (not shown) were found to be very similar in structure and composition to the precipitation regimes defined here, although the relative frequency of occurrence (RFO) of the four regimes does vary from year to year.

The mean FADs for each of the four regimes are shown in Fig. 3. The order of the panels in this figure is in terms of the RFO of each regime, which is shown in the top-left-hand corner of each panel. Because of its trivial nature the no-precipitation regime is not plotted; however, for completeness the RFO of this zeroth regime is displayed on the right-hand side of each panel. It is evident that for the dataset used here-hourly data for four wet seasons (NDJFMA) - this regime has the second highest frequency of occurrence (about 38\%), highlighting the well-known fact that even on the scale 

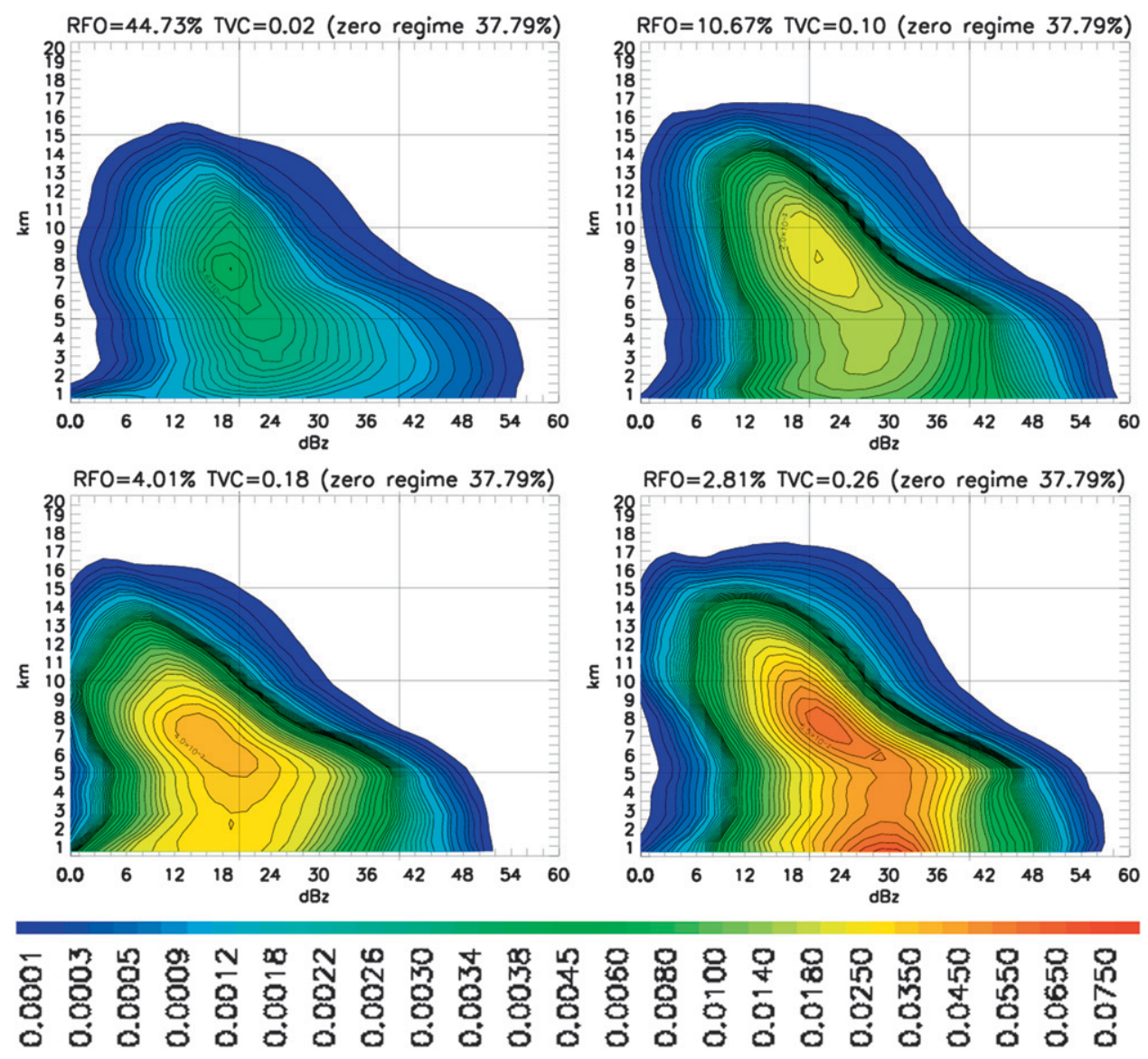

FIG. 3. The four precipitation regimes defined by the K-means algorithm. Regimes are ordered (most to least) by their RFO. The RFO of the zeroth regime (i.e., the regime containing time periods that have no precipitation over the entire radar domain) is listed for completeness. The contours show bin counts divided by total observed grid points.

of the radar footprint used here (about 300-km diameter) there are many times with no precipitation. Most of those occur in the transition season early and late in the wet-season definition used here (see section 4). Another important regime characteristic displayed in Fig. 3 is the TVC of the radar signal introduced in the previous section. The TVC provides a useful first indication for the differences between regimes. TVC increases steadily from $2 \%$ for the most frequent precipitation regime to $26 \%$ for the least frequent regime, indicating that in the Darwin region the tropical atmosphere exhibits regimes with small precipitation coverage significantly more frequently than those with large precipitation coverage.

Below follows a brief description of each of the regimes displayed in Fig. 3. In describing the regimes "low" reflectivities will refer to values between 0 and 20 $\mathrm{dB} Z$, "medium" reflectivities will refer to values between 20 and $40 \mathrm{~dB} Z$, and "high" reflectivities will refer to values between 40 and $60 \mathrm{~dB} Z$. Those ranges are indicated in the figures by vertical lines. To further aid conceptualization of the precipitation regimes Constant Altitude Plan Position Indicator (CAPPI) examples of each regime are shown in Fig. 4 for both 2.5 and $10 \mathrm{~km}$ in the vertical. These specific examples are representative of each regime and were chosen by finding those histograms with the minimum Euclidean distance from the cluster mean histograms displayed in Fig. 3. These CAPPI examples confirm that the regimes are well differentiated by TVC and the distribution of reflectivity values.

Regime 1 is the dominant weather condition occurring over Darwin during the four wet seasons analyzed. It occurs approximately $45 \%$ of the time. The precipitation associated with this regime is weak and shallow. It has the lowest frequency of reflectivity values above $40 \mathrm{dBZ}$ and a maximum echo-top height up to $2 \mathrm{~km}$ shallower when compared with the other regimes. The TVC for this regime is $2 \%$, which is an order of magnitude smaller than the next closest regime. The low TVC value and absence of a bright band at the freezing level (approximately $5 \mathrm{~km}$ ) indicates that the convection has a low spatial/ 

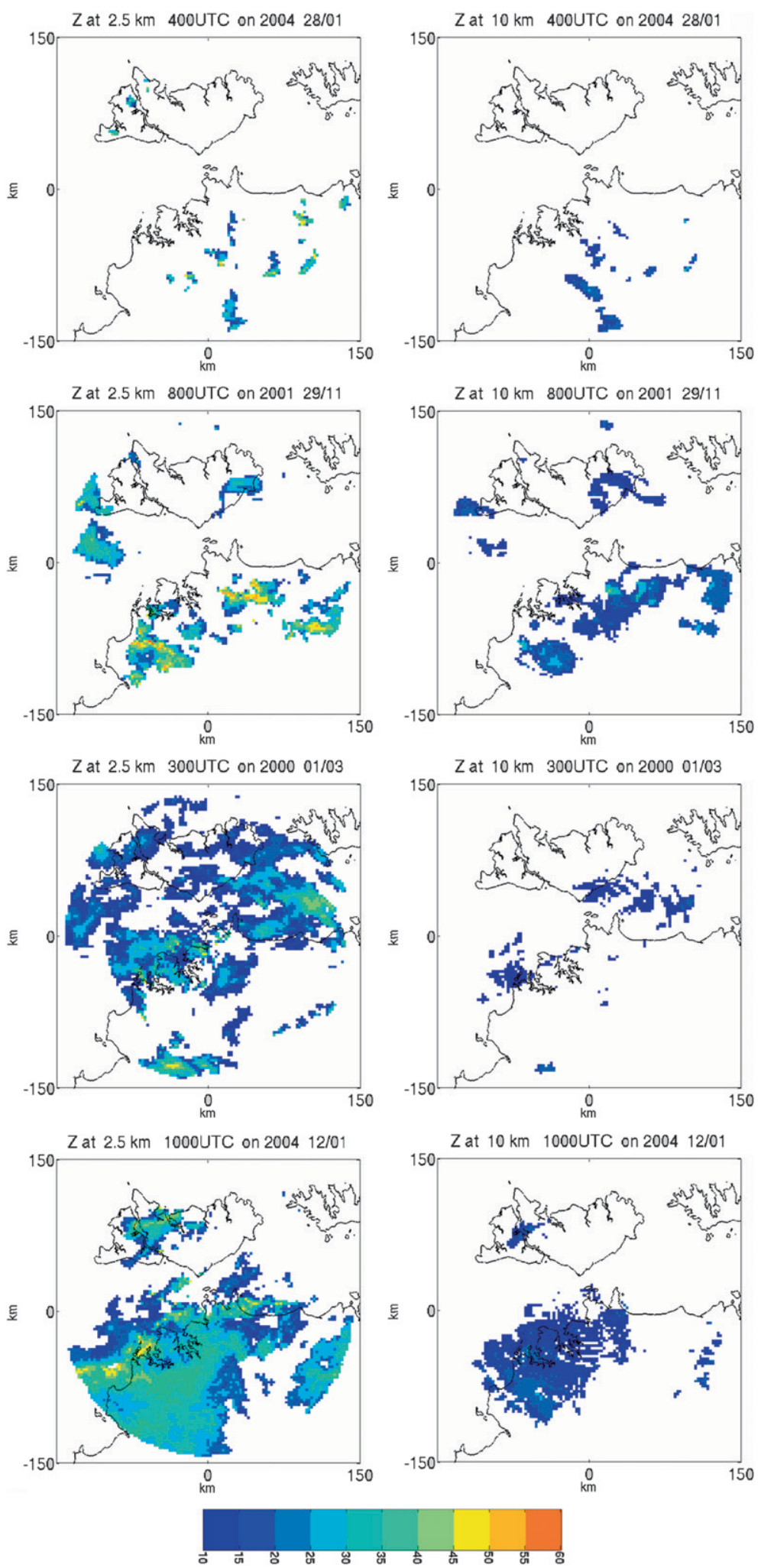

FIG. 4. CAPPI examples deemed representative of (top to bottom) the four precipitation regimes. Examples are given for both (left) 2.5 and (right) $10 \mathrm{~km}$ in the vertical. 
volume coverage and is therefore probably patchy and contains only a small proportion of stratiform precipitation. Average convective and stratiform rain contributions for each regime will be analyzed below to further investigate this hypothesis. On average, higher rain rates occur over the continent than over the ocean in this regime (cf. Fig. 5). This is an important feature of regime 1 and will be investigated further below.

Regime 2 has a RFO of approximately $10 \%$. The TVC of this regime is an order of magnitude greater than in regime 1 indicating that the precipitation covers a much larger volume of the radar domain. In this regime the maximum echo-top height extends to just over $16 \mathrm{~km}$ and there is a greater frequency of high reflectivity values at low levels, indicating that the convection is of greater intensity than was found for regime 1 . It should be noted that regime 2 actually has the highest reflectivity values of all four regimes. Another defining feature of regime 2 is the increased occurrence of medium reflectivity values $(20-40 \mathrm{dBZ})$ from the lowest level to about $13 \mathrm{~km}$, with the greatest frequency occurring between 6 and $12 \mathrm{~km}$.

As was the case in regime 1 , the absence of a strong brightband signal near $5 \mathrm{~km}$ is indicative of precipitation that is likely to be predominately convective, which will be further investigated in this section.

Regimes 3 has a RFO of approximately $4 \%$. A significant increase can be seen in both TVC and echo coverage at low levels. The low occurrence of high reflectivity values below $5 \mathrm{~km}$ and the decreased echotop height indicates that the convective component of this regime is weaker than the convection associated with regime 2. Oceanic convection tends to be shallower and have lower maximum reflectivity than continental convection (Toracinta et al. 2002; Nesbitt et al. 2000), the possibility that regime 3 could be influenced by precipitating systems with oceanic origins will be investigated in section 4 . The large increase in frequency of low and medium reflectivity values is likely due to the presence of significant areas of stratiform precipitation and would explain the relatively high TVC for this regime; evidence of stratiform precipitation can be seen in the form of a bright band at approximately $5 \mathrm{~km}$ for medium reflectivity values. However, as the regime is an average of many time periods and contains both stratiform and convective precipitation, the bright band is less pronounced than would normally be the case for purely stratiform states. Further evidence of the stratiform component of this regime will be provided in this section.

Regime 4 is the rarest of all the regimes and has a RFO of under $3 \%$. In contrast to regime 3 there is a large occurrence of high reflectivity values below $5 \mathrm{~km}$.
The maximum echo-top height for this regime is higher than in regime 3 and is even slightly higher than was found for regime 2 . The spread of reflectivity values at $15 \mathrm{~km}$ is larger than in regime 2 and extends to much higher reflectivity values than those found in regime 3 . Regime 4 has the largest TVC and by far the greatest frequency of medium reflectivity values, indicating the presence of large amounts of stratiform precipitation with greater vertical extent than the stratiform precipitation seen in regime 3 . The bright band in regime 4 is more pronounced than was seen in regime 3 and can be seen up to approximately $46 \mathrm{~dB} Z$. Another defining feature of regime 4 is the large frequency of medium reflectivity at the lowest levels, particularly between reflectivity values of 24 and $36 \mathrm{dBZ}$.

Figure 5 shows the average rain rate in millimeters per hour for the four regimes. For each time period used in the study (approximately 13500 ) the $2.5-\mathrm{km}$ reflectivity data was converted to rain rate maps using the relationship $Z=305 R^{1.36}$ (Bringi et al. 2001), where $Z$ is the absolute reflectivity and $R$ is the rain rate in millimeters per hour. Averaged rain-rate maps for each regime were then created by summing the $n$ rain-rate maps associated with each regime and dividing by $n$. The $R_{\text {total }}$ is displayed in the top-right-hand corner of each figure, calculated by summing the rain rates over the entire radar domain and dividing by the number of valid grid points, thereby giving some measure (spatially averaged) of the rain rate of each regime. The rainfall distribution in Fig. 5a shows that on average more rainfall occurs over land than over the ocean for regime 1 ; this is especially true over the Tiwi Islands. As expected this regime has the lowest rain rate of all the regimes, approximately $0.3 \mathrm{~mm} \mathrm{~h}^{-1}$ averaged over the entire domain.

Figure $5 \mathrm{~b}$ supports the assertion that regime 2 contains convection initiated by convergence due to landsea breezes; a slightly higher rain rate can be seen orientated parallel to the coastline of the mainland. Slightly higher rain rates can also be seen over the ocean in the west of the radar domain. Analysis of the diurnal cycle of rain rate for regime 2 (not shown) helps explain the patterns seen in Fig. 5b. During the afternoon precipitation occurs preferentially over land areas, while during the night and early morning, precipitation occurs over the ocean. The higher rain rates over the ocean in the west of the domain in Fig. 5b are due almost exclusively to precipitation occurring at night. Although $R_{\text {total }}$ of regime 2 is approximately 4 times that of regime 1 (cf. 1.1 and 0.3 ), its RFO is approximately $1 / 4$ times that of regime 1 (cf. approximately $11 \%$ for regime 2 and $45 \%$ for regime 1). Thus regimes 1 and 2 contribute approximately equal amounts of rainfall to the region. 

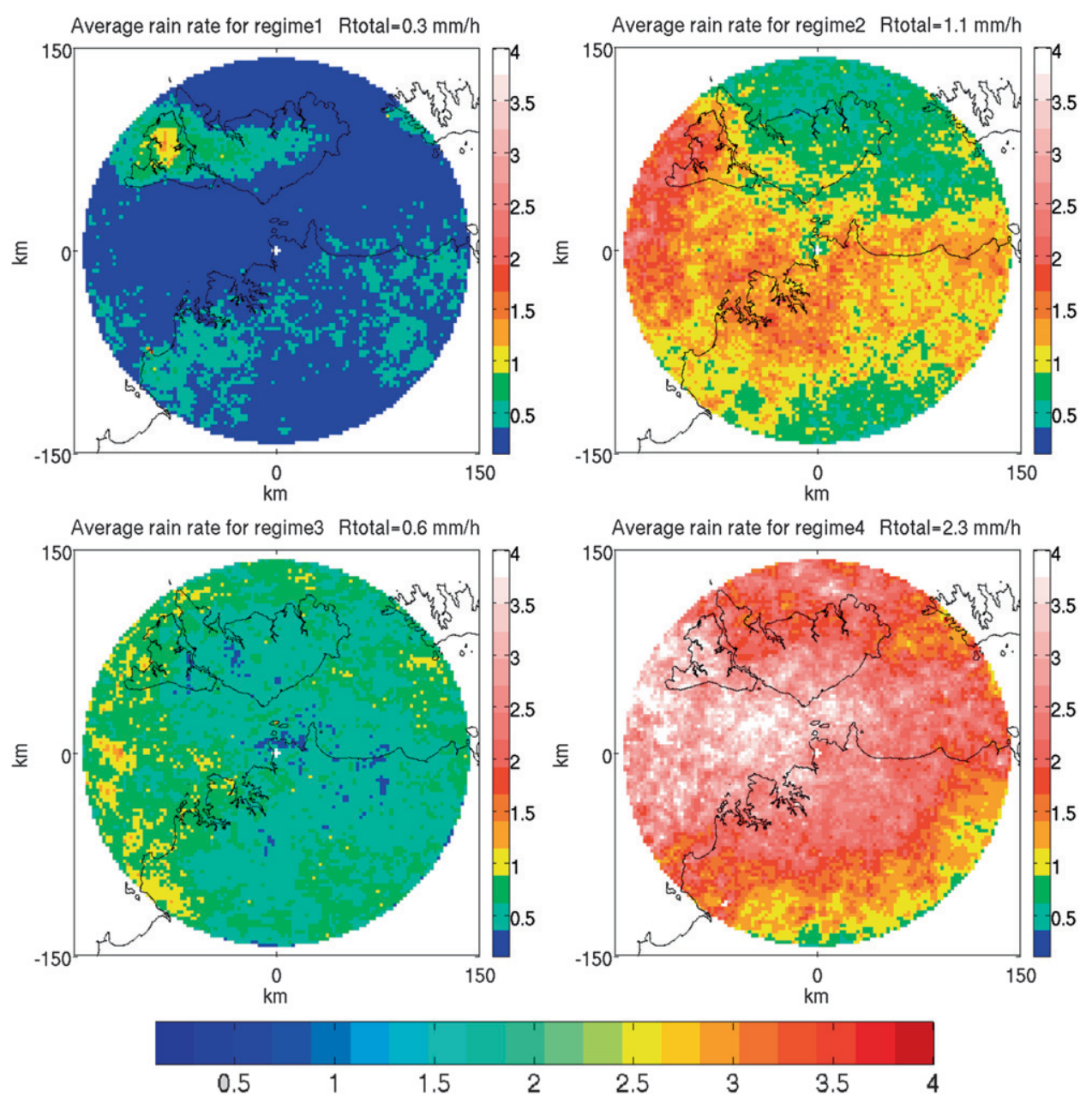

FIG. 5. Average rainfall rates for the four precipitation regimes in $\mathrm{mm} \mathrm{h}^{-1}$.

Figure $5 \mathrm{c}$ shows that in regime 3 rainfall is fairly constant throughout the domain of the radar, with perhaps a slight increase in rainfall over the ocean to the west. An interesting feature of this regime is that $R_{\text {total }}$ is much lower than was found for regime 2, despite the fact that the volume coverage of hydrometeors is much larger for regime 3 than regime 2 . This might be explained by the finding that the convection associated with regime 3 is weaker than the convection associated with regime 2.

Figure $5 \mathrm{~d}$ shows that in regime 4 high rainfall rates occur preferentially over the ocean. As expected, $R_{\text {total }}$ for this regime is the largest of all four regimes, owing to the large amounts of stratiform precipitation and intense convection found within this regime.

To further test the spatial distribution of the precipitation within the regimes a land-ocean mask was applied and the rain intensities over land and ocean were calculated for each regime. The land (ocean) rain in- tensity is defined as the sum of the rainfall over the land (ocean) divided by the number of land (ocean) points. Table 1 shows that the rain intensity is greater over land in regime 1 . Regime 2 has approximately the same rain intensity over land and ocean, as discussed above this regime contains contributions from convection occurring over land (during the afternoon) and over the ocean (during the night). Both regimes 3 and 4 have greater rain intensity over the ocean than the land.

Figure 6 shows the average rain rate for each regime split into convective and stratiform components. The rain fields measured with the C-Pol radar have been disaggregated into convective and stratiform areas using the algorithm described by Steiner et al. (1995). The only modification to this was to utilize the polarimetric rain estimates obtained with the C-Pol radar using algorithms described by Bringi et al. (2001, 2004). These rain estimates were then converted to an effective reflectivity using a simple $Z-R$ relation $\left(Z_{\text {eff }}=305 R^{1.36}\right)$ 
TABLE 1. Rain intensity of each regime split into land and ocean contributions $\left(\mathrm{mm} \mathrm{h}^{-1}\right)$.

\begin{tabular}{lcccc}
\hline \hline Regime & 1 & 2 & 3 & 4 \\
\hline Land & 0.3518 & 1.0824 & 0.5844 & 2.0265 \\
Ocean & 0.2252 & 1.1261 & 0.6656 & 2.6141 \\
\hline
\end{tabular}

in order to apply the Steiner et al. algorithms. This had the benefit of the classification being less sensitive to variations in measured reflectivity associated with drop size distributions, attenuation of the sampled reflectivity, and hail contamination as well as being consistent with the simple mapping of the observed field. The convective and stratiform rain was then summed over the radar domain (a 300-km-diameter circle) to calculate the contributions of convective and stratiform rain areas to the total rain accumulation. Figure 6 indicates that regime 1 is predominately a convective regime, with stratiform precipitation contributing $24 \%$ to the overall rain rate. Regime 2 is also predominately convective in nature; however, the stratiform component of this regime has increased to $35 \%$. Regime 3 contains the most stratiform precipitation relative to convective precipitation; $64 \%$ of the precipitation in regime 3 is stratiform in nature. Regime 4 also contains a large proportion of stratiform precipitation (53\%); however, the convective component of this regime is quite large, almost as high as the total rain rate for regime 2 .

In summary, analysis of the CFADs and convectivestratiform rain rates leads to the conclusion that regime 1 contains shallow, patchy, and relatively weak con- vection. Furthermore, the average rain-rate map for regime 1 and land-ocean rain intensities indicates that the convection associated with regime 1 preferentially occurs over land areas. Regime 2 was found to be a relatively strong convective regime that precipitates preferentially over the land during the day and over the ocean at night. The convection in this regime has greater intensity and scale than the convection in regime 1 , with a greater proportion of the convection likely initiated by land-sea breezes. The convective nature of this regime was confirmed by the relatively large proportion of convective rain as seen in Fig. 6 and absence of a brightband signature in Fig. 3. Regime 3 was found to contain a mixture of relatively weak convection and large amounts of stratiform precipitation. The temporal evolution of regimes (not shown), indicates that regime 3 is likely to occur from maturing systems associated with regimes 2 and 4 . Regime 4 was found to be a mixture of relatively strong convection with large amounts of stratiform precipitation.

Having discovered some of the basic characteristics of the regimes the next section aims to shed further light on their characteristics by studying their seasonal and diurnal cycles, and their link to the monsoon.

\section{Some characteristics of the precipitation regimes}

The previous section has objectively identified four (five if the no precipitation is included) major convective regimes in the Darwin region. The four nontrivial

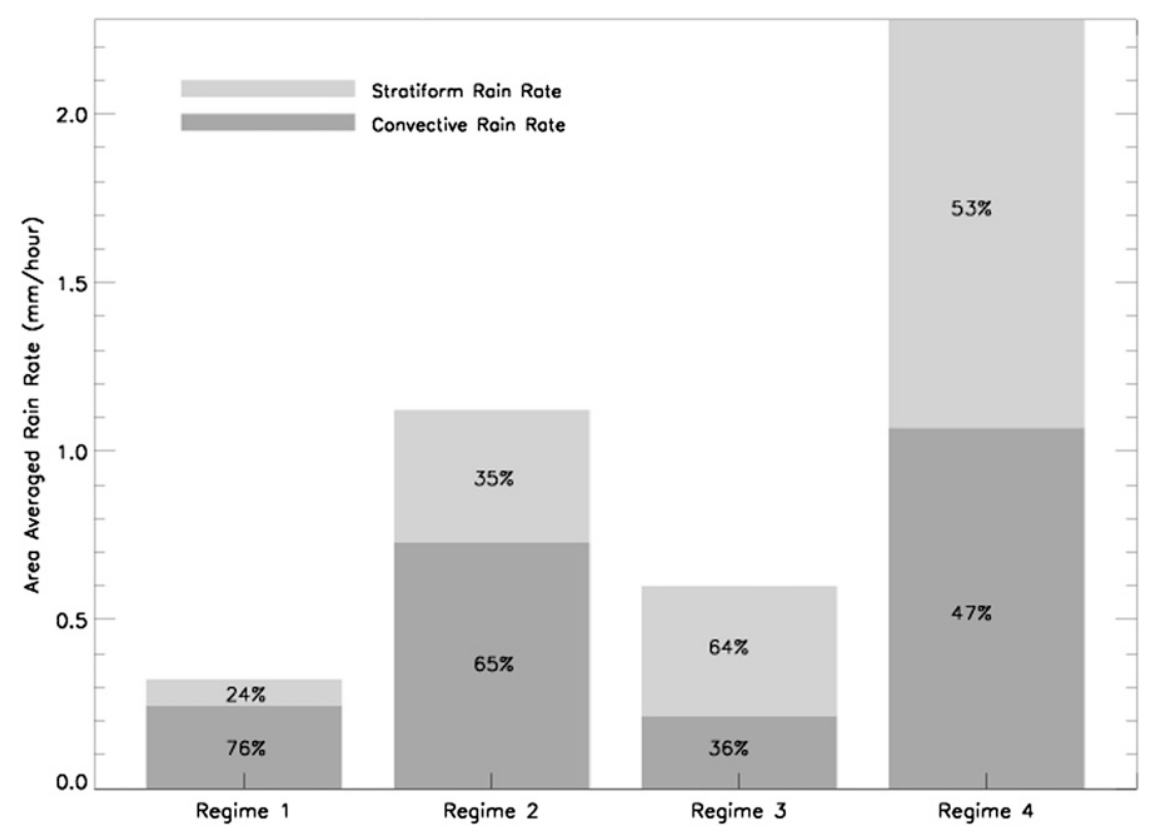

FIG. 6. Area-averaged stratiform and convective rain rates for the four precipitation regimes. 
regimes show characteristic distributions of radar reflectivity both in the horizontal and vertical on the scale of a typical weather radar footprint. While it was possible to link the regimes to known features of tropical convection, their link to large-scale features of the tropics as they affect the Darwin region remain to be elucidated. This section is aimed at establishing links between the regimes identified in the previous section and the known features of the tropical atmosphere. First their response to the main forced modes of the atmosphere, namely the seasonal and diurnal cycles are investigated. This is followed by establishing the regime characteristics as a function of monsoon activity in the Darwin region.

\section{a. The seasonal cycle}

In the Darwin region the seasonal mode is dominated by the Australian summer monsoon, which dominates the region's weather usually from the second half of December into early March (Drosdowsky 1996). Active monsoon periods are interlaced with monsoon breaks. The monsoon period is bordered by a buildup and decay period on the order of 1-2 months each, while the rest of the year has a distinct dry-season character.

Here the signature of the seasonal cycle on the convective regimes is investigated by studying the seasonal variation of their frequency of occurrence. Figure 7 shows the relative frequency of occurrence of the five regimes (including the no-precipitation regime) as a function of month. The no-precipitation regime (regime 0 ) shows the expected seasonal cycle with a minimum in January, the month with the largest influence of the Australian summer monsoon, and maxima in the months of March and April, those in which the Darwin region transits into the dry season. The RFO of the patchy convection regime (regime 1), is remarkably constant throughout November-February with a decline in occurrence in March and April. The stronger convective regimes (regimes 2-4) show an increase in their RFO from November to January, a decline in February and are virtually absent in March and April. The most notable change from November to January occurs for regimes 3 and 4, confirming the impression gained in the previous section that these regimes probably have a more monsoonal character (Steiner et al. 1995). This provides a further indication that the objectively identified precipitation regimes may represent physically relevant states of the atmosphere around Darwin, a claim that will be further investigated below.

\section{b. The diurnal cycle}

Figure 8 shows the diurnal cycle of the RFO of the four precipitating regimes. The RFO for each regime has been normalized including the no-precipitation regime. The diurnal cycles of the four precipitation regimes appear to be separated into two distinct patterns. Regimes 1 and 2 (top panels) have a peak in their RFO in the late afternoon, while regimes 3 and 4 (bottom panels) show a peak in the late at night and in the early hours of the morning. The two distinct patterns seen in the diurnal cycles may indicate a physical difference between the precipitation regimes. One possible explanation for this difference would be that the ratio of land to oceanic convection is different for the different regimes (as seen in Table 1). Convection tends to peak during the late afternoon/early evening over land, while over the oceans convection tends to peak during the early morning (Yang and Slingo 2001; Gray and Jacobson 1977; Mapes and Houze 1993). However any conclusion based on the diurnal cycle alone is problematic, Mapes and Houze (1993) have shown that the diurnal cycle of cold cloud clusters depends on the size of the cluster and Yang and Slingo (2001) showed that the diurnal signal over land often extends to the adjacent oceans. Furthermore Nesbitt and Zipser (2003) found that MCS rainfall over land peaks in the early morning. As regimes 3 and 4 both have high TVC values it is likely that MCS occurring over land are also associated with regimes 3 and 4.

Apart from the late afternoon maximum, regime 1 is equally likely to occur at any other hour of the day. This may be indicative for the fact that while land heating strongly influences regime 1 , this relatively weak convection may well occur over the ocean and at other times of the day. Earlier it was postulated that regime 2 has a larger proportion of convection initialed by sea breezes, if this is the case, the minimum in the diurnal cycle for regime 2 (around 10 am) may well be due to the lack of the sea breeze at this time. Regime 2 also displays a secondary peak in the early hours of the morning, which is likely due to the precipitation that occurs over the ocean in the western part of the domain.

\section{c. Regime occurrence and monsoon activity}

Another well-known indicator for the character of convection in the Darwin area is the synoptic meteorology of the Australian summer monsoon. A simple but effective definition of monsoon activity is wind direction in the lower troposphere, with westerly winds indicative for an active phase of the monsoon, while easterly winds dominate the break as well as pre- and postmonsoon periods (Drosdowsky 1996). Convection tends to be shallower and more representative of that found over oceans during periods when a westerly wind places Darwin in an oceanic air mass. Easterly wind 


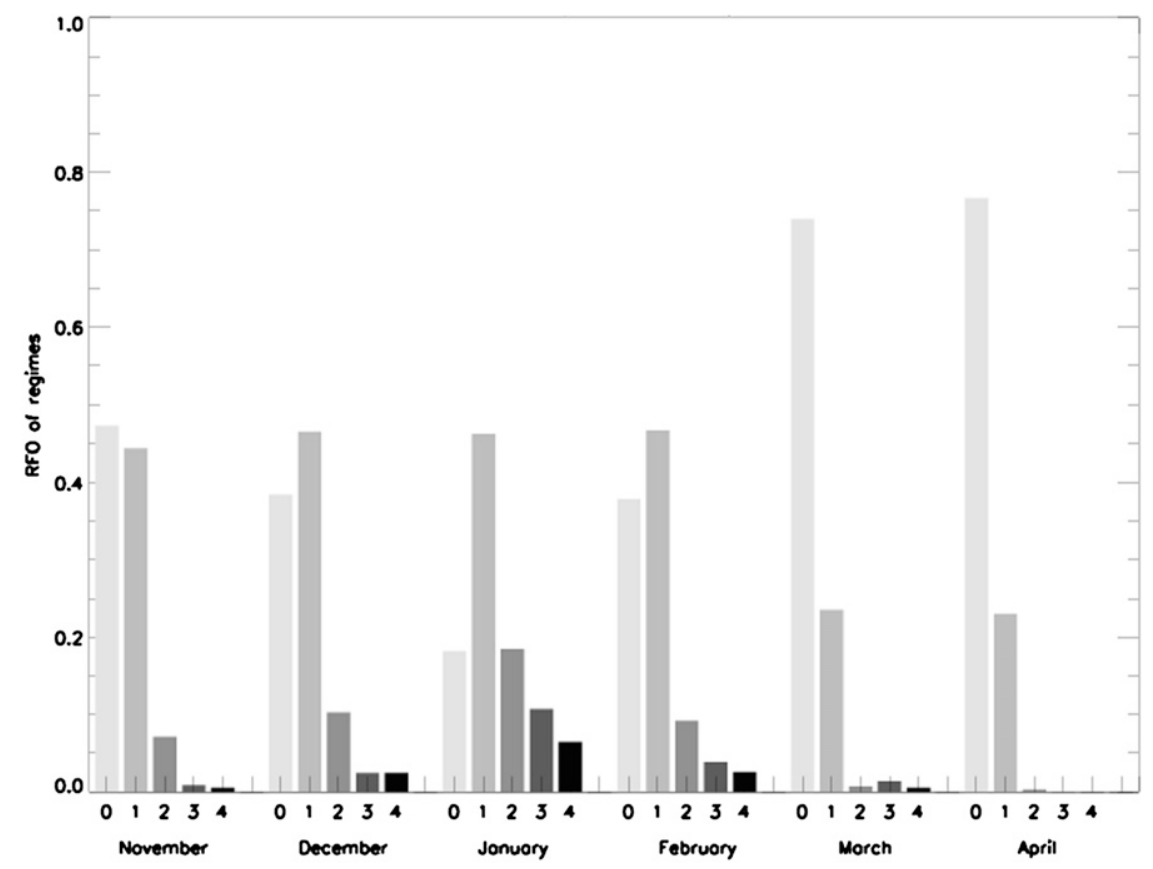

FIG. 7. Seasonal cycle of the precipitation regimes showing the RFO of each regime, for the months November-April.

conditions bring air from northeast Australia to the region and the convection is often more representative of that occurring over continents. Here the concept of an east-west split in wind direction that previously proved useful in other investigations (e.g., Keenan and Carbone 1992) is utilized with the aim of determining if the wind direction has any influence on the occurrence of the four precipitation regimes defined. The purpose of the analysis is not to find a clear delineation between the regimes, but rather to compare how the precipitation regimes found using cluster analysis compare to traditional methods. For the analysis, wind data from the Darwin airport at $700 \mathrm{hPa}$ is defined as easterly or westerly if the wind direction has any component in those directions. The frequencies of occurrence of wind direction within each regime are shown in Fig. 9.

It is evident from the figure that there are major differences between regimes in their relationship with wind direction and hence monsoon activity. Regimes $0-2$ show a predominance in their occurrence during times when the wind is coming from the east (break conditions), during break conditions precipitating cloud systems are known to be typical of those with continental origin (Steiner et al. 1995). The no-precipitation regime shows the strongest association with easterly flow. As indicated in Fig. 7 this regime occurs most frequently in the monsoon buildup and decay phases of the season. Those periods are associated with steady easterly flow, which explains the high frequency of occurrence of easterlies when this regime is found. Although regime 2 is predominately an easterly regime, it contains more westerly flow than regime 1 . This may help to explain why regime 2 was found to have to be a mixture of oceanic and land convection. In contrast, regimes 3 and 4 are most strongly associated with westerly conditions; westerly flow is typical for active monsoon periods, which have previously been shown to be associated with convection of maritime character (Keenan and Carbone 1992).

While simple, analyzing the predominant wind direction in each of the precipitation regimes has further supported the findings of the previous sections, that the precipitation regimes identified in this study are physically sensible. While probably following intuition, this constitutes a nontrivial finding, as there is no physical a priori information that supports that division entering into the cluster algorithm. The fact that the four (five) regimes could be objectively identified and match physical characteristics of convection rather well enables their use for further studies of convective systems in the Darwin area and possibly beyond.

\section{Precipitation versus ISCCP-based cloud regimes}

The previous section has established that the convective regimes identified in this study for the Darwin area exhibit two distinctly different patterns of their diurnal cycles and that the objectively defined regimes 

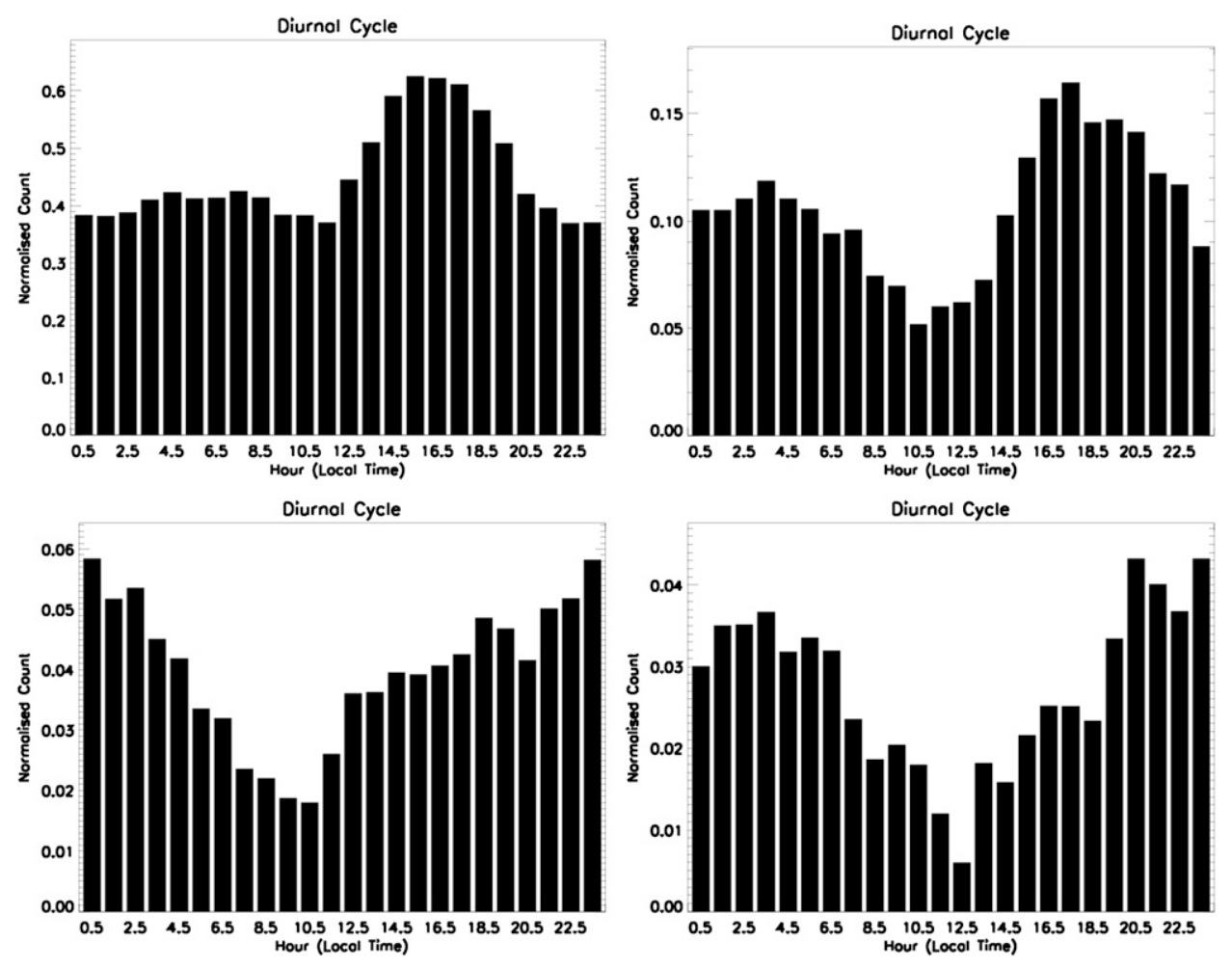

FIG. 8. Diurnal cycle of the four precipitation regimes (in local time), the RFO has been normalized including the zeroth regime.

are able to naturally separate when analyzing the synoptic-scale winds. Furthermore, the strength of the convection and the presence and size of the stratiform precipitation component all constitute information contained in the convective regimes. This enables the use of these regimes for further studies of tropical convection and its interaction with the large-scale circulation. Given this and the fact that this work was partially motivated by the success of finding tropical regimes based on cloud properties retrieved from satellites, it appears worthwhile to investigate the relationship of the six ISCCP-based tropical cloud regimes of Rossow et al. (2005) with the "precipitation-based" regimes identified here. This will provide some insight into the precipitation structure (in a statistical sense) of the cloud regimes, which in turn have already been shown to have links to tropical circulation features (Rossow et al. 2005; Höglund 2005).

To set the context for further discussion a brief summary of the six ISCCP weather states is given in this section; for a detailed discussion of the regimes the reader is referred to Rossow et al. (2005). Based on their cloud signature, three of the six regimes can be described as suppressed with respect to the occurrence of deep convection, while the other three have been identified as convectively active. Of the active regimes the deep anvil cloud (CD) regime exhibits a large coverage with optically thick clouds, most likely a mix of thick anvils and convective towers, while the convective cirrus (CC) regime is dominated by a large coverage with cirrus clouds of small-to-medium optical thickness. Probably the weakest (in terms of convection) of the three convectively active regimes is the mixed cloud (MIX) regime, termed because it likely consists of a mixture of shallow, congestus, and deep convection without significant anvil and cirrus coverage. The suppressed thin cirrus (STC) regime is categorized as a suppressed regime and is dominated by a large coverage with thin cirrus clouds. As shown by Rossow et al. (2005) this regime occurs close to convection without containing significant amounts of convective clouds in the area over which the ISCCP histogram is calculated. The suppressed shallow cloud regime (SSCL) regime has high frequencies of occurrence for clouds with high cloudtop pressure (low tops) and is interpreted as consisting in most part of suppressed shallow clouds with a low total cloud cover as is typically found in shallow cumulus cloud fields. The suppressed shallow cloud regime with high cloud cover ( $\mathrm{SSCH}$ ) is also dominated by low clouds, but generally shows a much higher total 


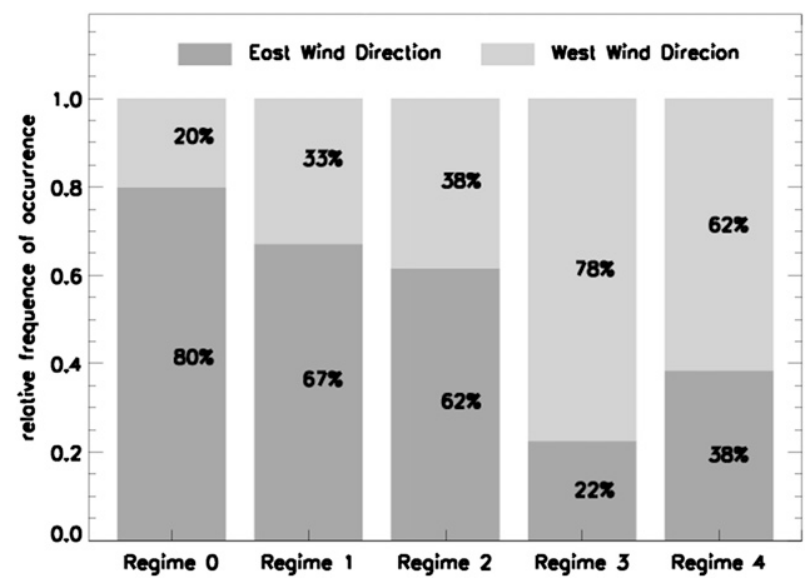

FIG. 9. RFO of the four precipitation and zeroth regimes for a given wind direction (defined as either easterly or westerly).

coverage with clouds indicative of stratocumulus clouds. Stratocumulus is almost never observed in the Darwin area resulting in an extremely low frequency of occurrence of the $\mathrm{SSCH}$ satellite regime in the study region. To avoid sampling biases this regime was excluded from further analysis.

As in previous studies (Höglund 2005) one ISCCP weather state per day is defined for each ISCCP grid box. As Darwin straddles several ISCCP grid boxes, sensitivity studies to the choice of grid point were carried out, but showed little sensitivity with regard to the main conclusions. Hence, the analysis presented here uses four ISCCP grid boxes each having an area of $280 \times 280 \mathrm{~km}^{2}$ located roughly between $10^{\circ}-15^{\circ} \mathrm{S}$ and $128.5^{\circ}-133^{\circ} \mathrm{E}$. The relationship of the ISCCP-based cloud regimes to the precipitation regimes of this study, which are identified hourly, is investigated by calculating the mean frequencies of occurrence of each precipitation regime on all days that fall into a particular ISCCP regime. Note that the C-Pol radar detects precipitation-sized particles and is unlikely to observe cirrus and nonprecipitating clouds.

Figure 10 shows the RFO of the five regimes of this study for each ISCCP-based cloud regime. The cloud regimes are sorted as in Rossow et al. (2005) from what is considered the most convectively active regime $(\mathrm{CD})$ to the most suppressed regime (SSCL). Several noteworthy features are evident in Fig. 10. The occurrence of no precipitation (regime 0 ) increases steadily from CD to SSCL. This supports the intuitive interpretation used in earlier work defining the ISCCP-based cloud regimes as convectively active versus suppressed. Of the suppressed regimes, SSCL clearly shows the least occurrence of deep convective regimes (regimes 2-4) and is mostly characterized by the relatively shallow pre- cipitation regime 1 . There is a steady increase in the deep convective regimes (regimes 2-4) for the MIX and CC regimes, mostly at the expense of the no-precipitation regime. Nevertheless, both these cloud regimes remain dominated by the regimes with low precipitation coverage (regimes 1 and 2). The only cloud regime with a significant occurrence of the regimes with high precipitation coverage (regimes 3 and 4 ) is the CD regime. In this regime the frequency of occurrence of no precipitation at all has dropped to around $10 \%$ indicating the frequent occurrence of precipitation in that regime. In earlier studies (Rossow et al. 2005; Höglund 2005) it was found that the ISCCP-based CD regime occurs predominantly in active phases (i.e., convergence zones) of large-scale tropical circulation features such as the Madden-Julian oscillation (MJO) or the Australian summer monsoon. This and the identification of the $\mathrm{CD}$ regime as the only regime containing high frequencies of regimes 3 and 4 leaves the intriguing possibility that what is thought of as "typical" maritime precipitating convection is in fact a convective type that is enabled and supported only by larger-scale circulation features.

An in-depth study into the precipitation characteristics of the ISCCP regimes is beyond the scope of this investigation; however, for completeness a simplistic calculation is performed to determine the average rain rate per hour of each ISCCP regime. As the rain rates for the ISCCP regimes have been derived from data obtained from the region surrounding Darwin, they are not intended to be applied to the tropics as a whole. The rain rate for each of the ISCCP regimes was calculated by multiplying the rain rate for each precipitation regime (Fig. 5) by the frequency with which the radar regimes occur within the given ISCCP regime (Fig. 10). These results are displayed in Table 2 and show that the rain rates for the ISCCP regimes is highest for the most convective regime $\mathrm{CD}$, and decreases corresponding to convective intensity, with the suppressed regimes having the lowest rain rates. It is interesting to note that the CC and the MIX regimes both have approximately the same rain rates. Although simplistic, associating an average rain rate with each of the ISCCP regimes can be thought of as a first step in creating/assessing parameterizations for numerical modeling. For example Fig. 10 and Table 2 show that from a precipitation point of view regimes CC and MIX are quite similar; therefore, it may be useful to create parameterizations for tropical weather conditions based on the ISSCP regimes (which represent distinct and different states of the tropical atmosphere that can distinguish the four main classes found here), using the radar regimes to estimate of the total rainfall and latent heating associated with each regime. 


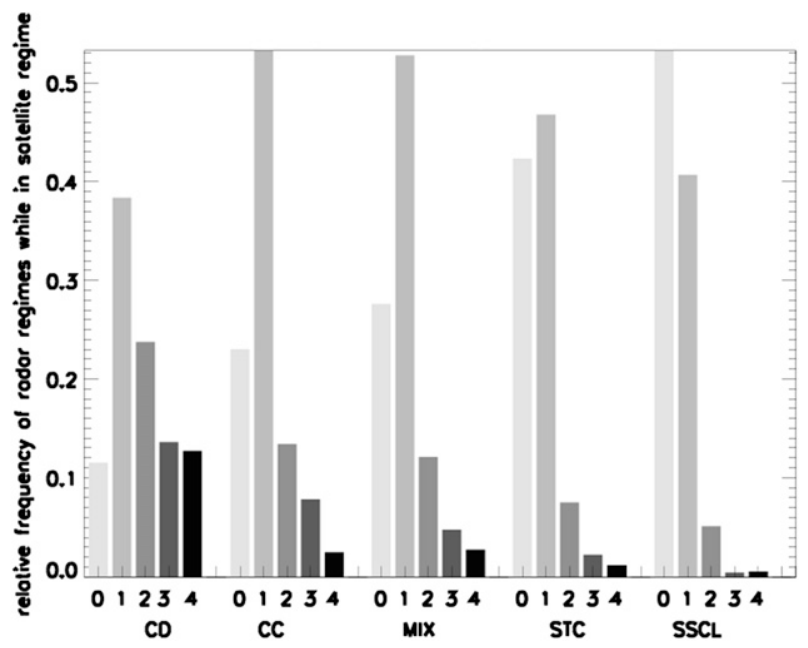

FIG. 10. Comparison between satellite and radar regimes. RFO as a function of radar regime, for the satellite regimes defined by Rossow et al. The SSCH satellite regime has not been included because of an extremely low frequency of occurrence.

\section{Concluding remarks}

This study has investigated if the three-dimensional structure of precipitation in the Darwin area as measured by a scanning polarimetric weather radar can be objectively classified into regimes. It has furthermore posed the question if those regimes have physical connections to the known features of the tropical atmosphere in the region. A cluster algorithm was applied to two-dimensional histograms of reflectivity with height derived from quasi-instantaneous radar volumes. After excluding a regime with virtually no radar returns in the volume (a "no-precipitation" regime) the analysis revealed the existence of four precipitation regimes in the Darwin region:

- Regime 1- a patchy convective regime of medium intensity and low area coverage. This regime occurs most frequently in the afternoon and during break conditions.

- Regime 2-a strong convective regime with relatively small area coverage. This regime has a peak in the diurnal cycle in the afternoon and a secondary peak in the early morning.

- Regime 3-a weak convective regime with large area coverage and large stratiform regions. This regime occurs most frequently during the late night/early morning and during monsoon conditions.

- Regime 4-a strong convective regime with large area coverage and large stratiform regions. This regime has a peak in the diurnal cycle in the late nightearly morning and has links to monsoon conditions.
TABLE 2. Calculated average rain rate of each ISCCP regime $\left(\mathrm{mm} \mathrm{h}^{-1}\right)$.

\begin{tabular}{ccccc}
\hline \hline CD & CC & MIX & STC & SSCL \\
\hline 0.8007 & 0.4325 & 0.3981 & 0.2729 & 0.1994 \\
\hline
\end{tabular}

The overall statistics of the occurrence of these regimes once again highlights that even during convectively active periods such as the Australian summer monsoon, the spatial coverage with precipitation is overall small (cf. Figs. 2 and 3).

Linking the regimes identified by the algorithm to known features such as the seasonal and diurnal cycle and a simple description of monsoon activity through wind direction has confirmed their different physical character. Regimes 1 and 2 show signs of a strongly landinduced diurnal cycle with an afternoon peak and occur throughout the wet season. They are largely associated with winds from the east, typical for monsoon buildup and decay as well as monsoon break conditions, during which convection is often observed to be of continental character. Regimes 3 and 4 have their largest frequency of occurrence in January-the peak of the monsoon season. Their diurnal variation peaks at night or early morning hours and they are largely associated with westerly winds, as is typical for monsoon conditions.

Combining the precipitation regimes with tropical cloud regimes previously identified from ISCCP data reveals interesting relationships between the two. Arguably there exist probably three (maybe four) broad precipitation regimes across the five ISCCP-based regimes present at Darwin. The suppressed shallow cloud regime (SSCL) is dominated by the no-precipitation and the patchy convection regimes. The stronger convection regimes, in particular, the maritime ones do not feature strongly in this cloud regime. While still dominated by the patchy convection regimes, the convective cirrus (CC) and mixed (MIX) cloud regimes and, to a lesser extent, the suppressed thin cirrus (STC) regime show larger frequencies of occurrence of the stronger precipitation regimes. The fraction of maritime versus continental precipitation regimes shifts steadily to more and more maritime with increasing convective character as identified in the cloud regimes themselves. By far the most active and most "maritime" regime is the convective deep anvil cloud regime (CD). About $50 \%$ of the time in this cloud regime one of the three strong convective precipitation regimes is present and this is the only regime where the strong maritime regime occurs with almost equal frequency to its weaker counterpart. The occurrence of no precipitation or patchy convection in this cloud regime is significantly reduced compared to any other cloud regime, showing the 
significantly different character of this regime. Given the aforementioned strong links of the $\mathrm{CD}$ cloud regime to large-scale circulation features (Rossow et al. 2005; Höglund 2005) further investigations into the precipitation characteristics using the regimes definitions derived here seem warranted.

The objective identification of precipitation regimes from radar data and the establishment of some of their physical characteristics open several avenues for further research. Their simplest application is in the objective identification of the overall character (i.e., mostly maritime versus mostly continental) and intensity (i.e., strong versus weak). Such characterization obviously already exists and is used regularly, but has so far largely been based on subjective criteria and/or the use of wind direction to identify convective character. The use of radar data as suggested here is both objective and more directly based on the precipitation features themselves. An obvious question for future research is how general the regimes found at Darwin are for the wider tropical region. Applying the techniques proposed here to other radar locations in the tropics, such as Kwajalein (Cetrone and Houze 2006), would be a natural extension of this study. Another possibility would be the application to data collected by the TRMM precipitation radar. It would also be interesting to investigate if regimes defined in a similar way to those used here could helpful in objectively determining some of the "building blocks" used in the stretched building block conceptual model described by Mapes et al. (2006).

The regimes identified here represent the statistical characteristics of convection at the spatial scale of the radar footprint (roughly $300 \mathrm{~km}^{2}$ ). Studying the regime identification as a function of scale is therefore another possible extension of this work. As has been highlighted here, combining the precipitation regime information with data from different types of instruments is another promising avenue of research. The brief example of the ISCCP-based cloud regimes emphasizes the potential of such investigations. The presence of a large suite of instruments deployed by the U.S. Department of Energy's ARM program (Ackerman and Stokes 2003) as well as recently conducted field studies such as TWPICE (May et al. 2005) make Darwin the ideal location for such studies. On a longer time scale, objectively defined precipitation regimes may also prove useful when studying local changes in rainfall patterns due to changes in synoptic conditions and seasonal weather patterns (e.g., El Niño). A similar analysis could be performed over a much larger time scale to determine how or if the precipitation regimes change with time and determine any possible links with climate change. Finally it would be very interesting to investigate if models, in particular those that resolve deep convection, are able to reproduce the observed regimes when forced with realistic large-scale conditions. Recent field data will enable such studies in the near future.

Acknowledgments. We thank Dr. Courtney Schumacher for many insightful discussions. We are grateful for support from the U.S. Department of Energy under Grants DE-FG02-04ER63823, DE-FG02-03ER63533, and LANL-23662-001-013T as part of the Atmospheric Radiation Measurement Program.

\section{REFERENCES}

Ackerman, T. P., and G. M. Stokes, 2003: The Atmospheric Radiation Measurement Program. Phys. Today, 56, 38-44.

Alexander, M. J., P. T. May, and J. H. Beres, 2004: Gravity waves generated by convection in the Darwin area during the Darwin Area Wave Experiment. J. Geophys. Res., 109, D20S04, doi:10.1029/2004JD004729.

Anderberg, M. R., 1973: Cluster Analysis for Applications. Academic Press, 359 pp.

Boccippio, D., W. Petersen, and D. Cecil, 2005: The tropical convective spectrum. Part I: Archetypal vertical structures. $J$. Climate, 18, 2744-2769.

Bringi, V. N., G.-J. Huang, V. Chandrasekar, and T. D. Keenan, 2001: An areal rainfall estimator using differential propagation phase: Evaluation using a C-band radar and a dense gauge network in the Tropics. J. Atmos. Oceanic Technol., 18, $1810-1818$.

_ T. Tang, and V. Chandrasekar, 2004: Evaluation of a new polarimetrically-based $Z-R$ relation. J. Atmos. Oceanic Technol., 21, 612-622.

Carbone, R. E., J. W. Wilson, T. D. Keenan, and J. M. Hacker, 2000: Tropical island convection in the absence of significant topography. Part I: Life cycle of diurnally forced convection. Mon. Wea. Rev., 128, 3459-3480.

Carey, L. D., and S. A. Rutledge, 2000: The relationship between precipitation and lightning in tropical island convection: A C-Band polarimetric radar study. Mon. Wea. Rev., 128, 2687-2710.

Cetrone, J., and R. A. Houze Jr., 2006: Characteristics of tropical convection over the ocean near Kwajalein. Mon. Wea. Rev., 134, 834-853.

Crook, N. A., 2001: Understanding Hector: The dynamics of island thunderstorms. Mon. Wea. Rev., 129, 1550-1563.

Demott, C. A., and S. A. Rutledge, 1998: The vertical structure of TOGA COARE convection. Part I: Radar echo distributions. J. Atmos. Sci., 55, 2730-2747.

Drosdowsky, W., 1996: Variability of the Australian summer monsoon at Darwin: 1957-1992. J. Climate, 9, 85-96.

Gray, W. M., and R. W. Jacobson, 1977: Diurnal variation of deep cumulus convection. Mon. Wea. Rev., 105, 1171-1188.

Gunn, B. W., J. L. McBride, G. J. Holland, T. D. Keenan, and N. E. Davidson, 1989: The Australian summer monsoon circulation during AMEX phase II. Mon. Wea. Rev., 117, 2554-2574.

Hamilton, K., R. A. Vincent, and P. T. May, 2004: Darwin Area Wave Experiment (DAWEX) field campaign to study gravity wave generation and propagation. J. Geophys. Res., 109, D20S01, doi:10.1029/2003JD004393.

Höglund, S., 2005: Clouds in Darwin and their relation to largescale conditions. M.S. thesis, Department of Applied Physics 
and Mechanical Engineering, Division of Physics, Lulea University of Technology, Lulea, Sweden, 61 pp. [Available online at http://epubl.luth.se/1402-1617/2005/169/LTU-EX05169-SE.pdf.]

Holland, G. J., J. L. McBride, R. K. Smith, D. Jasper, and T. D. Keenan, 1986: The BMRC Australian Monsoon Experiment. Bull. Amer. Meteor. Soc., 67, 1466-1472.

Houze, R. A., Jr., 1987: Mesoscale organization and cloud microphysics in a Bay of Bengal depression. J. Atmos. Sci., 44, 18461867.

, 1993: NimboStratus. Cloud Dynamics, R. Dmowska and J. R. Holton, Eds., Vol. 53, Academic Press, 196-220.

1997: Stratiform precipitation in regions of convection: A meteorological paradox? Bull. Amer. Meteor. Soc., 78, 2179 2196.

— , and D. D. Churchill, 1984: Microphysical structure of winter monsoon cloud clusters. J. Atmos. Sci., 41, 3405-3411.

— C.-P. Cheng, C. A. Leary, and J. F. Gamache, 1980: Diagnosis of cloud mass and heat fluxes from radar and synoptic data. J. Atmos. Sci., 37, 754-773.

_ S. G. Geotis, F. D. Marks, D. D. Churchill, and P. H. Herzegh, 1981: Comparison of airborne and land-based radar measurements of precipitation during winter MONEX. J. Appl. Meteor., 20, 772-783.

Jakob, C., and G. Tselioudis, 2003: Objective identification of cloud regimes in the Tropical Western Pacific. Geophys. Res. Lett., 30, 2082, doi:10.1029/2003GL018367.

— - - a and T. Hume, 2005: The radiative, cloud, and thermodynamic properties of the major tropical western Pacific cloud regimes. J. Climate, 18, 1203-1215.

Kawashima, M., Y. Fujiyoshi, M. Oh, T. Honda, T. Kozu, T. Shimomat, and H. Hashiguchi, 2006: Overview of Doppler radar observations of precipitating cloud systems in Sumatera Island during the first CPEA campaign. J. Meteor. Soc. Japan, 84A, 33-56.

Keenan, T. D., and R. E. Carbone, 1992: A preliminary morphology of precipitation systems in tropical northern Australia. Quart. J. Roy. Meteor. Soc., 118, 283-326.

— monsoonal convection and associated stratiform precipitation. Mon. Wea. Rev., 121, 352-374.

—, J. McBride, G. Holland, N. Davidson, and B. Gunn, 1989: Diurnal variations during the Australian Monsoon Experiment (AMEX) phase II. Mon. Wea. Rev., 117, 2535-2552.

— K. Glasson, F. Cummings, T. S. Bird, J. Keeler, and J. Lutz, 1998: The BMRC/NCAR C-Band Polarimetric (C-POL) radar system. J. Atmos. Oceanic Technol., 15, 871-886.

— , and Coauthors, 2000: The Maritime Continent Thunderstorm Experiment (MC-TEX): Overview and some results. Bull. Amer. Meteor. Soc., 81, 2433-2455.

Lin, J., B. Mapes, M. Zhang, and M. Newman, 2004: Stratiform precipitation, vertical heating profiles, and the MaddenJulian oscillation. J. Atmos. Sci., 61, 296-309.

Mapes, B. E., 1993: Gregarious tropical convection. J. Atmos. Sci., 50, 2026-2037.

—, and R. A. Houze Jr., 1993: Cloud clusters and superclusters over the oceanic warm pool. Mon. Wea. Rev., 121, 1398-1415.

— wind divergence in regions of tropical convection. Mon. Wea. Rev., 133, 1808-1824.

— S. Tulich, J. Lin, and P. Zuidema, 2006: The mesoscale convection life cycle: Building block or prototype for largescale tropical waves. Dyn. Atmos. Oceans, 42, 3-29.
May, P. T., T. D. Keenan, D. S. Zrnić, L. D. Carey, and S. A. Rutledge, 1999: Polarimetric radar measurements of tropical rain at a 5-cm wavelength. J. Appl. Meteor., 38, 750-765.

_ J. H. Mather, G. Vaughan, C. Jakob, G. M. McFaquhar, K. Bower, and G. G. Mace, 2005: The Tropical Warm Pool International Cloud Experiment (TWP-ICE). Bull. Aust. Meteor. Oceanogr. Soc., 18, 109-110.

Neiman, P. J., G. A. Wick, F. M. Ralph, B. E. Martner, A. B. White, and D. E. Kingsmill, 2005: Wintertime nonbrightband rain in California and Oregon during CALJET and PACJET: Geographic, interannual, and synoptic variability. Mon. Wea. Rev., 133, 1199-1223.

Nesbitt, S. W., and E. Zipser, 2003: The diurnal cycle of rainfall and convective intensity according to three years of TRMM measurements. J. Climate, 16, 1456-1475.

,-- , and D. J. Cecil, 2000: A census of precipitation features in the Tropics using TRMM: Radar, ice scattering, and lightning observations. J. Climate, 13, 4087-4106.

$\longrightarrow$, R. Cifelli, and S. A. Rutledge, 2006: Storm morphology and rainfall characteristics of TRMM precipitation features. Mon. Wea. Rev., 134, 2702-2721.

Orlanski, I., 1975: A rational subdivision of scales for atmospheric processes. Bull. Amer. Meteor. Soc., 56, 527-530.

Pautet, P.-D., M. J. Taylor, A. Z. Liu, and G. R. Swenson, 2005: Climatology of short-period gravity waves observed over northern Australia during the Darwin Area Wave Experiment (DAWEX) and their dominant source regions. J. Geophys. Res., 110, D03S90, doi:10.1029/2004JD004954.

Rickenbach, T. M., and S. Rutledge, 1998: Convection in TOGA COARE: Horizontal scale, morphology, and rainfall production. J. Atmos. Sci., 55, 2715-2729.

Rossow, W. B., G. Tselioudis, A. Polak, and C. Jakob, 2005: Tropical climate described as a distribution of weather states indicated by distinct mesoscale cloud property mixtures. Geophys. Res. Lett., 32, L21812, doi:10.1029/2005GL024584.

Schiffer, R. A., and W. B. Rossow, 1983: The International Satellite Cloud Climatology Project (ISCCP): The first project of the World Climate Research Programme. Bull. Amer. Meteor. Soc., 64, 779-784.

Schumacher, C., and R. A. Houze Jr., 2003: Stratiform rain in the Tropics as seen by the TRMM Precipitation Radar. J. Climate, 16, 1739-1756.

- - , and I. Kraucunas, 2004: The tropical dynamical response to latent heating estimates derived from the TRMM Precipitation Radar. J. Atmos. Sci., 61, 1341-1358.

Steiner, M., R. A. Houze Jr., and S. E. Yuter, 1995: Climatological characterization of three-dimensional storm structure from operational radar and rain gauge data. J. Appl. Meteor., 34, 1978-2007.

Tao, W.-K., and Coauthors, 2006: Retrieval of latent heating from TRMM measurements. Bull. Amer. Meteor. Soc., 87, 1555-1572.

Toracinta, E. R., D. J. Cecil, E. J. Zipser, and S. W. Nesbitt, 2002: Radar, passive microwave, and lightning characteristics of precipitating systems in the Tropics. Mon. Wea. Rev., 130, 802-824.

Yang, G.-Y., and J. Slingo, 2001: The diurnal cycle in the Tropics. Mon. Wea. Rev., 129, 784-801.

Yuter, S. E., and R. A. Houze Jr., 1995: Three-dimensional kinematic and microphysical evolution of Florida cumulonimbus. Part II: Frequency distributions of vertical velocity, reflectivity, and differential reflectivity. Mon. Wea. Rev., 123, 1941-1963.

Zipser, E. J., D. J. Cecil, C. Liu, S. W. Nesbitt, and D. P. Yorty, 2006: Where are the most intense thunderstorms on Earth? Bull. Amer. Meteor. Soc., 87, 1057-1071. 